\title{
Tunable magnetic alignment between trapped exciton-polariton condensates
}

\author{
H. Ohadi, ${ }^{1}$ Y. del Valle-Inclan Redondo, ${ }^{1}$ A. Dreismann, ${ }^{1}$ Y. G. Rubo, ${ }^{2}$ F. \\ Pinsker, ${ }^{3}$ S. I. Tsintzos, ${ }^{4}$ Z. Hatzopoulos, ${ }^{4,5}$ P. G. Savvidis, ${ }^{4,6}$ and J. J. Baumberg ${ }^{1}$ \\ ${ }^{1}$ Department of Physics, Cavendish Laboratory, University of Cambridge, Cambridge CB3 0HE, United Kingdom \\ ${ }^{2}$ Instituto de Energías Renovables, Universidad Nacional Autónoma de México, Temixco, Morelos, 62580, Mexico \\ ${ }^{3}$ Clarendon Laboratory, University of Oxford, Parks Road, Oxford OX1 3PU, United Kingdom \\ ${ }^{4}$ Foundation for Research and Technology-Hellas, \\ Institute of Electronic Structure and Laser, 71110 Heraklion, Crete, Greece \\ ${ }^{5}$ Department of Physics, University of Crete, 71003 Heraklion, Crete, Greece \\ ${ }^{6}$ Department of Materials Science and Technology, \\ University of Crete, 71003 Heraklion, Crete, Greece
}

\begin{abstract}
Tunable spin correlations are found to arise between two neighboring trapped exciton-polariton condensates which spin-polarize spontaneously. We observe a crossover from an antiferromagneticto a ferromagnetic pair state by reducing the coupling barrier in real-time using control of the imprinted pattern of pump light. Fast optical switching of both condensates is then achieved by resonantly but weakly triggering only a single condensate. These effects can be explained as the competition between spin bifurcations and spin-preserving Josephson coupling between the two condensates, and opens the way to polariton Bose-Hubbard ladders.
\end{abstract}

The development of spin-charge lattice models for understanding strongly-correlated states of matter is a successful theme of modern quantum physics. This has driven the desire to model and probe complex condensed matter phenomena using highly-controlled systems, such as ultra-cold atoms [1], photons [2, 3], or superconducting junctions [4]. Exciton-polariton (polariton) lattices have emerged as an alternative system $[5,6]$ with unique properties. Due to their strongly dissipative and nonlinear nature, many-body polariton gases can reach steady states which are remarkably different from their equilibrium case [7]. Moreover, they have peculiar spin properties [8-10] and exhibit spontaneous magnetization (emitting circularly polarized light) above a critical bifurcation threshold [11], analogous to the weak lasing regime [12]. In this Letter we study the basic building block of a polariton spin lattice: two optically trapped spin-polarized condensates which are tunably coupled. We demonstrate that trapped out-of-equilibrium polariton condensates can exhibit Ising-like behavior related to spin bifurcations. The two condensate system investigated here is shown to correspond to one plaquette of a bosonic ladder [13], and allows demonstration of a crossover in the competition between Josephson coupling and spin bifurcation. These features have not been seen in any other system to date.

Polariton condensates are coherent many-body states [14-17], which can be confined in potentials [18-20] and interact with each other via Josephson junctions [21-24]. For a pair of interacting trapped spin-polarized condensates, their polarization states are expected to couple. However, the driven-dissipative and nonlinear nature of polariton condensates makes the underlying coupling mechanism considerably richer than that in the conventional Ising case, leading to exotic forms of magnetism where the orientation and strength of coupling is not determined by the sign of the interaction. (a)

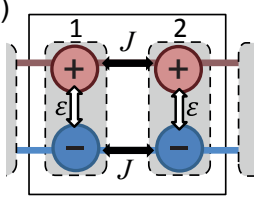

(b)

(c)
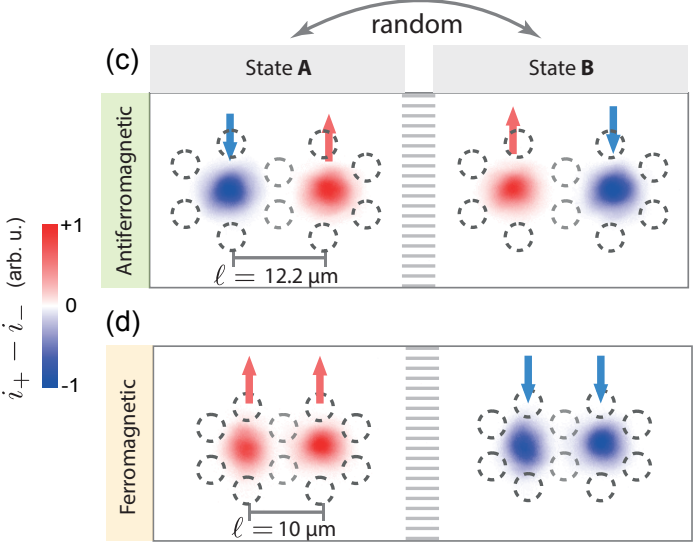

FIG. 1. (a) Coupling between condensates 1,2 controlled by Josephson tunneling $J$, spin-coupling $(+\leftrightarrow-)$ within condensates controlled by energy splitting $\varepsilon$. (b) Schematic double condensate trap from 10 pump beams. (c,d) Experimental spin states seen for (c) AF- and (d) F-coupled condensates. In each case two possible states exist, with actual state chosen randomly upon each realization.

We achieve tuning between ferromagnetic $(\mathrm{F})$ or antiferromagnetic (AF) coupling by directly modulating the tunneling barrier, adjusting either the height of the barrier or the separation between the condensates. We show how optical switching of the spin state of one condensate results in fast switching of the state of the neighboring condensate. Our result is a key step towards using trapped polariton condensates for the realization of interacting bosons in a driven-dissipative spinor Bose- 
Hubbard model [5, 7].

The Bose-Hubbard model with polaritons can be studied in other systems where, for example, the sample is etched to form micro-pillar arrays [6, 10, 24], metal films are deposited on the surface of the cavity [21, 25, 26], or surface acoustic waves are applied [27]. While the confinement potential is then separate from the condensate gain, the trapped exciton-polaritons studied in the present letter have the advantage that the confinement, even at a single site level, is versatile and can be adjusted on the fly. Particularly for larger arrays of condensates, this capability to tune the different barriers is vital. Moreover, the interaction with the reservoir particles is reduced, crucial for spin stability.

Exciton-polaritons (polaritons) are quasiparticles formed by the strong coupling of excitons in semiconductor quantum wells with photons in the microcavity in which they are embedded [28]. We create optically trapped polariton condensates $[20,29,30]$ by nonresonant linearly-polarized continuous wave (CW) excitation of a membrane microcavity [31]. Driven by their repulsive excitonic interactions, polaritons travel away from the pump region and feed the zero-momentum ground state at the center of the optical trap. Once the density exceeds the condensation threshold, a macroscopically coherent condensate forms in the trap center. Polaritons in quantum-well microcavities have two \pm 1 (spin up or down) projections of their total angular momentum along the structure growth axis, which correspond to right- and left-circularly polarized photons emitted by the cavity (of intensities $i_{ \pm}$).

Trapped polariton condensates spontaneously exhibit a high degree of circular polarization, or magnetization $M=s_{z}=\left(i_{+}-i_{-}\right) /\left(i_{+}+i_{-}\right)$, above a critical spinbifurcation threshold as a result of energy and dissipation splitting of their linear polarizations [11]. We operate above this spin-bifurcation threshold, which means that the trapped condensate is spin-polarized in either spin-up $|\uparrow\rangle$ or spin-down $|\downarrow\rangle$ states. These spin-polarized condensates emit nearly circularly polarized $(|M|>85 \%)$ light, which can be measured using conventional polarimetry. In each realization we excite the sample for $200 \mu$ s and measure the condensate $M_{n}(t)$, where $n \in\{1,2\}$ denotes the left and right condensate [32]. The optical excitation is patterned using a spatial light modulator (SLM) into the shape of a double-hexagon as shown by dashed circles in Fig. 1(b-d) such that a spin-polarized condensate is formed at the center of each hexagon. The middle 'barrier' pump spots [orange in Fig. 1(b)] between the two traps are weaker than the outer spots (intensity ratio $\simeq 75 \%$ ) to allow inter-trap tunneling of polaritons.

We can spatially squeeze or stretch the traps and change the condensates separation without changing the barrier pump intensity. To achieve this the barrier pump spots of the double-hexagon trap are fixed while shifting the location of the other spots. When the separation of the condensates maxima is greater than $\ell_{c}=13.6 \mu \mathrm{m}$, the two condensates independently pick a spin-up or
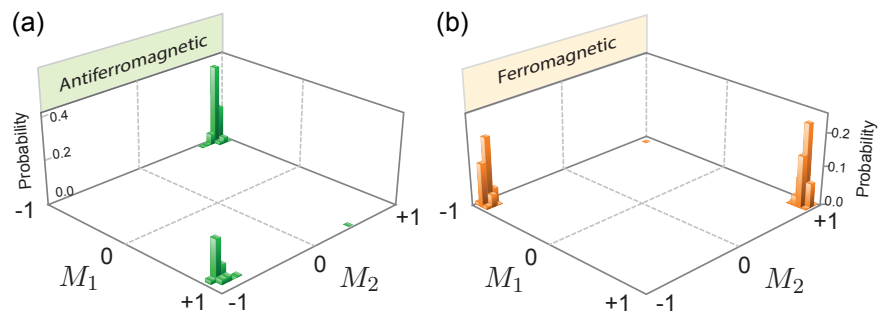

FIG. 2. 2D histogram of correlations in measured double condensate spins $\left(M_{1}, M_{2}\right)$ over 1000 realizations in the (a) antiferromagnetic and (b) ferromagnetic coupling regimes.

spin-down state. However, when this is decreased to $\ell=0.90 \ell_{c}$, we observe AF coupling, where the condensates spontaneously collapse into either $|\uparrow \downarrow\rangle$ or $|\downarrow \uparrow\rangle$ states in each realization [Fig. 1(c)]. Further decreasing the separation to $0.74 \ell_{c}$, we observe $\mathrm{F}$ coupling where the condensates pick either of $|\uparrow \uparrow\rangle$ or $|\downarrow \downarrow\rangle$ states randomly in each realization [Fig. 1(d)]. As in the case of a single condensate [11], these states remain stable for many seconds at $5 \mathrm{~K}$, and do not depend on the position on the sample, the geometrical pattern of the pump spots or the power above the spin-bifurcation threshold.

For each set of trapping conditions, 1000 realizations are created and for each we measure $M$ for the two condensates to perform a statistical analysis of the condensate-pair spin correlation. Each polarizationresolved realization is recorded for $200 \mu \mathrm{s}$ by a camera, allowing us to map the $2 \mathrm{D}$ histogram of $\left(M_{1}, M_{2}\right)$ and resolving $\mathrm{F}$ and AF situations (Fig. 2). Absolute correlations of $|C|>0.99$ are found for the condensate spins in both coupling regimes. Increasing the condensate separation to $\ell_{c}$ reduces this correlation to 0.09 , confirming that condensate spins then become uncoupled. It is important to note that we observe partial phase coherence between the condensates in both $\mathrm{AF}$ and $\mathrm{F}$ regimes, and the condensates are at equal energies [33]).

To accurately map the magnetic phase diagram of the system the influence of barrier on condensate spin correlation is investigated. Instead of changing the separation of the condensates by changing the trap geometry, we vary their barrier potential. For this the intensity of the barrier pump spots [orange, Fig. 1(a)] is changed. This allows finer control over the coupling interaction than changing the separation of the condensates, which is discretized due to single-pixel shifting of the SLM. To better observe the correlations, we induce spin flips by increasing the spin noise via spatially broadening the pump spots, which increases the overlap of the condensates and the pump. This increases the spontaneous spin-flip rate of the condensates (here set to $\sim 10 \mathrm{flips} / \mu \mathrm{s},[34]$ ), allowing faster and more reliable correlation measurements within the stability time of our setup. Since the energy blueshift from pump-injected excitons is proportional to the pump intensity [29], the ratio of the intensity of barrier pump spots to the other spots, $u_{r}$, is a reliable mea- 

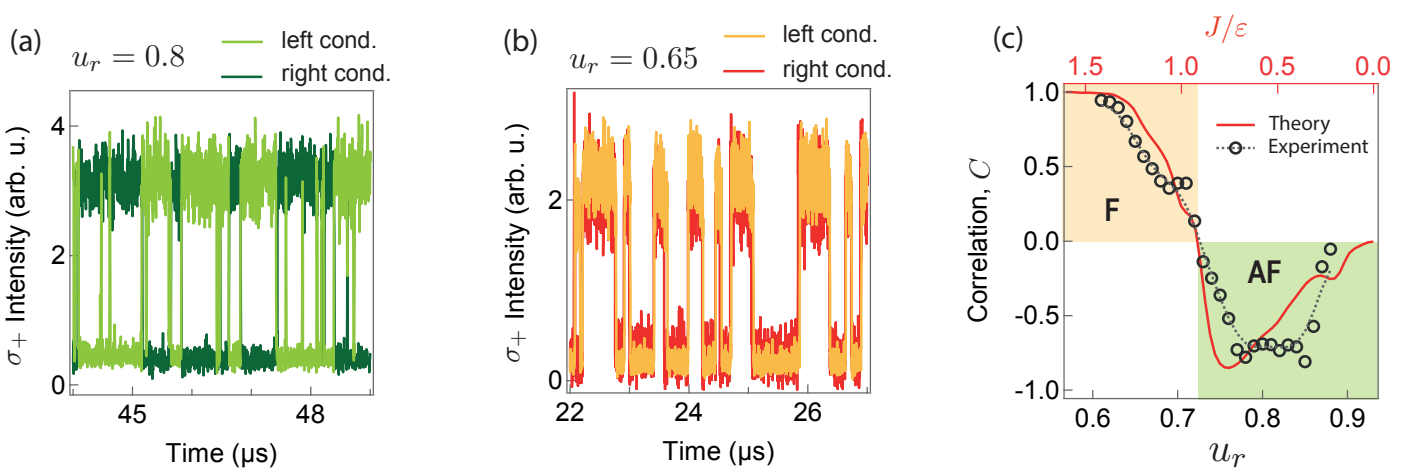

FIG. 3. (a,b) Snapshot in time of left and right condensate $\sigma_{+}$intensities for (a) $u_{r}=0.80$ and (b) $u_{r}=0.65$, showing spontaneous correlated flipping of both condensates spins in (a) AF and (b) F regimes. (c) Magnetic phase diagram, with measured correlation $C$ between magnetization of condensates 1,2 as a function of barrier $u_{r}$ (circles, dotted line is guide for the eye). Simulations give the correlation $C$ as a function of $J / \varepsilon$ (red line)

sure of the relative barrier height. The blueshift above the condensate energy at the saddle point of the barrier is $u_{r} U_{0}$, where $U_{0} \simeq 200 \mu \mathrm{eV}$. Selecting the right-circularly polarized $\left(\sigma_{+}\right)$emission, we spatially resolve the left and right condensates recording their intensities at each $u_{r}$ for $2 \mathrm{~ms}$ using photomultipliers. A typical trace for $u_{r}=0.8$ [Fig. 3(a)] shows that the double condensates flip randomly between the two AF states with a switching time limited by our measurement resolution $(\sim 5 \mathrm{~ns})$. Reducing $u_{r}$ to 0.65 shows now flipping between two F-states [Fig. 3(b)]. For each barrier potential we record 20 traces (each lasting $75 \mu \mathrm{s}$ ) and calculate the average correlation of the condensates spins, plotting this as a function of $u_{r}$ [Fig. 3(c)]. We observe a clear transition from the ferromagnetic to antiferromagnetic state at $u_{r} \simeq 0.72$. Increasing $u_{r}$ further results in zero coupling.

The uncoupling of the condensates when increasing their separation $\ell / \ell_{c}=0.9 \rightarrow 1.0$ (while the intensity of the shared pump spots remains constant) implies that the shared reservoir between two condensates does not play a significant role here. Our trapped condensates form with $\bar{k}=0(\Delta k=0.4 \mathrm{rad} / \mu \mathrm{m})$, where the transverseelectric and transverse-magnetic splitting vanishes [35]. As a result the optical spin-Hall effect [36] is negligible in trapped condensates, with spin torque rates much smaller than tunnelling rates thus preserving spin during the Josephson process [37]. On the other hand, observation of phase coherence between the condensates signifies that the spin coupling must be mediated by a coherent mechanism, as described by our theory below.

Our description of above effects is based on the theory for a single trapped condensate [11], which is extended to include the Josephson coupling [38-40] between the two condensates. The order parameter for each excitonpolariton condensate is a two-component complex vector $\Psi_{n}=\left[\psi_{n+}, \psi_{n-}\right]^{\mathrm{T}}$ and $\psi_{n+}$ and $\psi_{n-}$ are the spin-up and spin-down wave functions. The components of the order parameter define the measurable condensate pseudospin $\mathbf{S}_{n}=(1 / 2)\left(\Psi_{n}^{\dagger} \cdot \boldsymbol{\sigma} \cdot \Psi_{n}\right)$, and the normalized spin vector $\hat{\mathbf{s}}_{n}=\mathbf{S}_{n} / S_{n}$, where $\sigma_{x, y, z}$ are the Pauli matrices. The or- der parameters evolve according to the driven dissipative equation

$$
\begin{aligned}
\mathrm{i} \frac{d \Psi_{n}}{d t}= & -\frac{\mathrm{i}}{2} g\left(S_{n}\right) \Psi_{n}-\frac{\mathrm{i}}{2}(\gamma-\mathrm{i} \varepsilon) \sigma_{x} \Psi_{n} \\
& +\frac{1}{2}\left[\left(\alpha_{1}+\alpha_{2}\right) S_{n}+\left(\alpha_{1}-\alpha_{2}\right) S_{n z} \sigma_{z}\right] \Psi_{n} \\
& -\frac{1}{2} J \Psi_{3-n} .
\end{aligned}
$$

Here $g\left(S_{n}\right)=\Gamma-W+\eta S_{n}$ is the pumping-dissipation balance, $\Gamma$ is the (average) dissipation rate, $W$ is the incoherent in-scattering (or 'harvest' rate), and $\eta$ captures the gain-saturation term [41]. This gain saturation depends on the total occupation of the condensate (treated more generally in Ref. [11]). $X$ (horizontal) and $Y$ (vertical) linearly-polarized single-polariton states are split in energy by $\varepsilon$ and dissipation rate by $\gamma$. The repulsive interaction constant for polaritons with the same spin is $\alpha_{1}$, and the interaction constant for polaritons with opposite spins is $\alpha_{2}$. Finally, $J>0$ is the extrinsic spin-preserving Josephson coupling between the left and the right condensate, which we have introduced here to account for coherent coupling.

It is important to note the fundamental differences between the spin coupling mechanism demonstrated here and that seen in closed systems such as atoms trapped in optical lattices [42, 43]. In equilibrium spin systems such as those described by the Ising model, the coupling is achieved via the minimization of the total energy. In the driven-dissipative system described here, the minimization of energy does not play a direct role since the system is out of equilibrium. Here the spin alignment is a direct result of spin bifurcation correlation, which itself is a product of pumping, dissipation and nonlinearity, and coherent exchange of particles between the two condensates.

We perform dynamical simulations to calculate spin correlations. We calculate the steady state of the coupled Eq. 1 for 10,000 realizations with random initial conditions [44] and plot the final circular polarization correlation of the two condensates at different Joseph- 
(a)

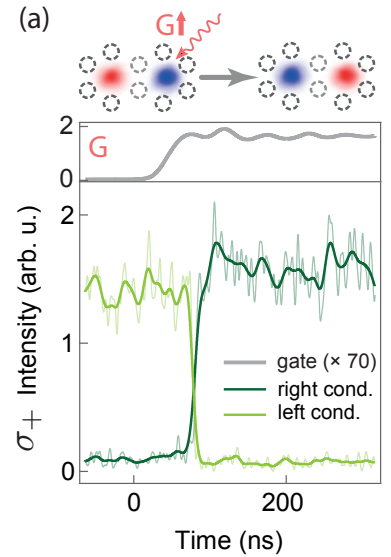

(b)

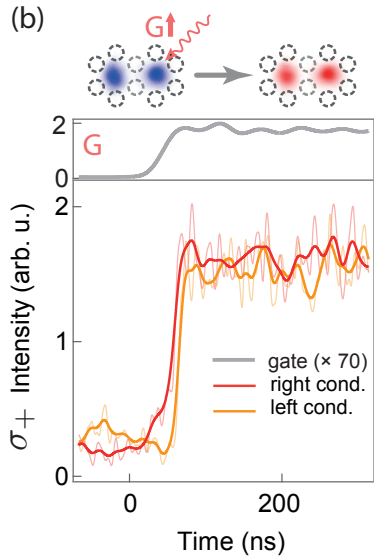

FIG. 4. (a) Spin switching for AF-coupled condensates. The $\sigma_{+}$-polarized gate is applied on the right condensate (shown above). The condensate-pair switches from $|\uparrow \downarrow\rangle$ to $|\downarrow \uparrow\rangle$. (b) Spin switching for F-coupled condensates. The condensatepair switches from $|\downarrow \downarrow\rangle$ to $|\uparrow \uparrow\rangle$. The top panels show the gate laser intensity profile $\mathrm{G}$. The gate laser is turned on at $t=0$.

son coupling rates [Fig. 3(c), red line]. For small Josephson amplitudes there is AF coupling of the condensate states. The stable AF configuration is characterized by $\psi_{2+}=-\psi_{1-}$ and $\psi_{2-}=-\psi_{1+}$, so that Eq. (1) is reduced to the two-component problem with renormalized splitting between $X$ - and $Y$-polarized states $\varepsilon^{\prime}=\varepsilon-J$. Correspondingly, the AF state loses stability at $\varepsilon=J$ when $\varepsilon^{\prime}$ changes sign. The AF state is first converted to the limit cycle motion by the Hopf bifurcation and then to pseudo-chaotic behavior with further increase of $J$. Simultaneously, the stable F configuration with $\psi_{2+}=\psi_{1+}$ and $\psi_{2-}=\psi_{1-}$ emerges for $J>\varepsilon$. This behavior is in good agreement with the experiment: we observe the AF coupling at high barrier heights where the tunneling rate between two condensates (Josephson coupling) is small and same-site coupling between spins dominates [Fig. 1(a), white arrows]. On the other hand when the barrier height is low, F-coupling is seen since the Josephson coupling dominates [Fig. 1(a), black arrows] [45]. Each interacting pair of condensates then forms a plaquette, and longer chains of condensates will form a bosonic ladder [13], which will in future be useful to probe with magnetic fields [46]. We note that paired condensates emit light at the same frequency, as it is also found experimentally.

The chaotic dynamics in the AF-F crossover region of the present system is different from the chaos in resonantly excited pair of exciton-polariton condensates studied before [47]. The trapped exciton-polariton condensates here are excited incoherently, yielding chaos in an autonomous dynamical system. The details of this chaos and its effects on the emission from the trapped conden-

sates will be studied elsewhere.

Finally, we demonstrate the resonant switching of the coupled spin states experimentally. We resonantly excite one of the condensates with a narrow linewidth $\mathrm{CW}$ diode laser, which we refer to as the gate $(G)$. The membrane microcavity allows resonant excitation from the back side of the cavity without requiring filtering of the laser backscatter. The gate laser as well as the pump can be switched on or off by acousto-optic modulators (AOM) with a rise time of $\sim 40 \mathrm{~ns}$. We resonantly excite the right condensate $20 \mu \mathrm{s}$ after we turn on the pump laser. The gate is applied on the right-hand condensate and is right-circularly polarized $\left(\sigma_{+}\right)$. Applying a cross-polarized gate switches the polarization state of the condensate, as previously shown [11]. Here, we observe that resonant switching of the spin of one condensate also switches the spin of the other coupled condensate, both in the antiferromagnetic and ferromagnetic regimes (Fig. 4). The condensates remain in the switched state after the gate laser is switched off, due to the bistable nature of the spin states. We note that the resolutionlimited condensate switching time reported here is an order of magnitude shorter than that of the gate, clearly showing that (as for single condensates [11]) the switching process is nonadiabatic. Here we are able to switch the coupled spin states by transiently injecting minority spins which are only $1 \%$ of the condensates majority spin. Our theoretical description reproduces the fast spin flips observed in the experiment when noise is injected into Eq. 1. Our theory also shows that in both AFand F-regimes, switching the spin state of condensate 1 also switches the spin of condensate 2 , both remaining switched after the pulse is turned off [48].

In conclusion, we demonstrate a tunable spin coupling mechanism for trapped polariton condensates. A transition from antiferromagnetic to ferromagnetic coupling is seen as the potential barrier between the condensates decreases. Our results correspond well to the interplay between spin-bifurcation and Josephson coupling in theory. Finally resonant switching of the spin states in both ferromagnetic and antiferromagnetic regimes is shown.

Acknowledgments-Authors acknowledge Andrew Ramsay, Dieter Jaksch and Tomi Johnson for fruitful discussions. This work was supported by EPSRC Grant No. EP/G060649/1, EP/L027151/1, EU Grant No. INDEX 289968, ERC Grant No. LINASS 320503, the Leverhulme Trust Grant No. VP1-2013-011, Spanish MEC (Grant No. MAT2008-01555), the Greek GSRT ARISTEIA Apollo program and Fundación La Caixa, and Mexican CONACYT Grant No. 251808. FP acknowledges financial support through a Schrödinger Fellowship at the University of Oxford and NQIT project (EP/M013243/1). The data corresponding to the figures in this paper can be found at https: //www.repository. cam.ac.uk/handle/1810/253679. 
[1] I. Bloch, J. Dalibard, and W. Zwerger, Rev. Mod. Phys. 80, 885 (2008).

[2] A. Szameit and S. Nolte, J. Phys. B: At. Mol. Opt. Phys. 43, 163001 (2010).

[3] M. Hafezi, S. Mittal, J. Fan, A. Migdall, and J. M. Taylor, Nat. Photon. 7, 1001 (2013).

[4] J. Q. You and F. Nori, Nature 474, 589 (2011).

[5] I. Carusotto and C. Ciuti, Rev. Mod. Phys. 85, 299 (2013).

[6] T. Jacqmin, I. Carusotto, I. Sagnes, M. Abbarchi, D. D. Solnyshkov, G. Malpuech, E. Galopin, A. Lemaître, J. Bloch, and A. Amo, Phys. Rev. Lett. 112, 116402 (2014).

[7] A. Le Boité, G. Orso, and C. Ciuti, Phys. Rev. Lett. 110, 233601 (2013).

[8] C. Leyder, M. Romanelli, J. P. Karr, E. Giacobino, T. C. H. Liew, M. M. Glazov, A. V. Kavokin, G. Malpuech, and A. Bramati, Nat. Phys. 3, 628 (2007).

[9] K. G. Lagoudakis, T. Ostatnický, A. V. Kavokin, Y. G. Rubo, R. André, and B. Deveaud-Plédran, Science 326, 974 (2009).

[10] V. G. Sala, D. D. Solnyshkov, I. Carusotto, T. Jacqmin, A. Lemaître, H. Terças, A. Nalitov, M. Abbarchi, E. Galopin, I. Sagnes, et al., Phys. Rev. X 5, 011034 (2015).

[11] H. Ohadi, A. Dreismann, Y. G. Rubo, F. Pinsker, Y. del Valle-Inclan Redondo, S. I. Tsintzos, Z. Hatzopoulos, P. G. Savvidis, and J. J. Baumberg, Phys. Rev. X 5, 031002 (2015).

[12] I. L. Aleiner, B. L. Altshuler, and Y. G. Rubo, Phys. Rev. B 85, 121301 (2012).

[13] M. Atala, M. Aidelsburger, M. Lohse, J. T. Barreiro, B. Paredes, and I. Bloch, Nat Phys 10, 588 (2014).

[14] H. Deng, G. Weihs, C. Santori, J. Bloch, and Y. Yamamoto, Science 298, 199 (2002).

[15] J. Kasprzak, M. Richard, S. Kundermann, A. Baas, P. Jeambrun, J. M. J. Keeling, F. M. Marchetti, M. H. Szymańska, R. André, J. L. Staehli, et al., Nature 443, 409 (2006).

[16] R. Balili, V. Hartwell, D. Snoke, L. Pfeiffer, and K. West, Science 316, 1007 (2007).

[17] J. Baumberg, A. Kavokin, S. Christopoulos, A. Grundy, R. Butté, G. Christmann, D. Solnyshkov, G. Malpuech, G. Baldassarri Höger von Högersthal, E. Feltin, et al., Phys. Rev. Lett. 101, 136409 (2008).

[18] E. Wertz, L. Ferrier, D. D. Solnyshkov, R. Johne, D. Sanvitto, A. Lemaître, I. Sagnes, R. Grousson, A. V. Kavokin, P. Senellart, G. Malpuech, and J. Bloch, Nat. Phys. 6, 860 (2010).

[19] G. Tosi, G. Christmann, N. G. Berloff, P. Tsotsis, T. Gao, Z. Hatzopoulos, P. G. Savvidis, and J. J. Baumberg, Nat. Phys. 8, 190 (2012).

[20] P. Cristofolini, A. Dreismann, G. Christmann, G. Franchetti, N. G. Berloff, P. Tsotsis, Z. Hatzopoulos, P. G. Savvidis, and J. J. Baumberg, Phys. Rev. Lett. 110, 186403 (2013).

[21] C. W. Lai, N. Y. Kim, S. Utsunomiya, G. Roumpos, H. Deng, M. D. Fraser, T. Byrnes, P. Recher, N. Kumada, T. Fujisawa, and Y. Yamamoto, Nature 450, 529 (2007).

[22] K. G. Lagoudakis, B. Pietka, M. Wouters, R. André, and B. Deveaud-Plédran, Phys. Rev. Lett. 105, 120403
(2010).

[23] G. Tosi, G. Christmann, N. G. Berloff, P. Tsotsis, T. Gao, Z. Hatzopoulos, P. G. Savvidis, and J. J. Baumberg, Nat. Comm. 3, 1243 (2012).

[24] M. Abbarchi, A. Amo, V. G. Sala, D. D. Solnyshkov, H. Flayac, L. Ferrier, I. Sagnes, E. Galopin, A. Lemaître, G. Malpuech, and J. Bloch, Nat. Phys. 9, 275 (2013).

[25] N. Y. Kim, C.-W. Lai, S. Utsunomiya, G. Roumpos, M. Fraser, H. Deng, T. Byrnes, P. Recher, N. Kumada, T. Fujisawa, and Y. Yamamoto, phys. stat. sol. (b) 245, 1076 (2008).

[26] N. Y. Kim, K. Kusudo, C. Wu, N. Masumoto, A. Löffler, S. Höfling, N. Kumada, L. Worschech, A. Forchel, and Y. Yamamoto, Nat. Phys. 7, 681 (2011).

[27] E. A. Cerda-Méndez, D. N. Krizhanovskii, M. Wouters, R. Bradley, K. Biermann, K. Guda, R. Hey, P. V. Santos, D. Sarkar, and M. S. Skolnick, Phys. Rev. Lett. 105, 116402 (2010).

[28] A. V. Kavokin, J. Baumberg, G. Malpuech, and F. P. Laussy, Microcavities (Oxford Univ. Press, Oxford, 2007).

[29] A. Askitopoulos, H. Ohadi, A. V. Kavokin, Z. Hatzopoulos, P. G. Savvidis, and P. G. Lagoudakis, Phys. Rev. B 88, 041308 (2013).

[30] A. Dreismann, P. Cristofolini, R. Balili, G. Christmann, F. Pinsker, N. G. Berloff, Z. Hatzopoulos, P. G. Savvidis, and J. J. Baumberg, Proc. Natl. Acad. Sci. U.S.A. 112, E1516 (2014).

[31] See Supplemental Material 1 at [url], which includes Refs. [49, 50], for details of the sample.

[32] See Supplemental Material 2 at [url] for real-space intensity profiles.

[33] See Supplemental Material 3 at [url], which includes Refs. [51, 52], for phase and energy measurements.

[34] See Supplemental Material 4 at [url] for pump-induced spin flips measurement.

[35] I. Shelykh, K. V. Kavokin, A. V. Kavokin, G. Malpuech, P. Bigenwald, H. Deng, G. Weihs, and Y. Yamamoto, Phys. Rev. B 70, 035320 (2004).

[36] A. Kavokin, G. Malpuech, and M. Glazov, Phys. Rev. Lett. 95, 136601 (2005).

[37] At $k=0.4 \mathrm{rad} / \mathrm{\mu m}$ the half-wavelength of the spin precession observed in Ref. 53 is $\sim 375 \mu \mathrm{m}$, which is more than 2 orders of magnitude larger than the condensate separations we have here.

[38] M. Wouters and I. Carusotto, Phys. Rev. Lett. 99, 140402 (2007).

[39] I. A. Shelykh, D. D. Solnyshkov, G. Pavlovic, and G. Malpuech, Phys. Rev. B 78, 041302 (2008).

[40] D. Read, Y. G. Rubo, and A. V. Kavokin, Phys. Rev. B 81, 235315 (2010).

[41] J. Keeling and N. G. Berloff, Phys. Rev. Lett. 100, 250401 (2008).

[42] J. Struck, C. Ölschläger, R. L. Targat, P. Soltan-Panahi, A. Eckardt, M. Lewenstein, P. Windpassinger, and K. Sengstock, Science 333, 996 (2011).

[43] D. Greif, T. Uehlinger, G. Jotzu, L. Tarruell, and T. Esslinger, Science 340, 1307 (2013).

[44] Similar to Ref. 11, the parameters used for 0D simulations are: $W=0.18 \mathrm{ps}^{-1} ; \Gamma=0.1 \mathrm{ps}^{-1} ; \varepsilon=0.04 \mathrm{ps}^{-1}$; $\gamma=0.2 \varepsilon ; \alpha_{1}=0.01 \mathrm{ps}^{-1} ; \alpha_{2}=-0.5 \alpha_{1} ; \eta=0.02 \mathrm{ps}^{-1}$. 
[45] We note that apart from most probable pure $\mathrm{F}$ and $\mathrm{AF}$ configuration, other attractors exist for the system (1) even away from the crossover region $J \approx \varepsilon$. In particular, the $\mathrm{F}$ configuration for $J>\varepsilon$ can be also achieved with the broken $1 \leftrightarrow 2$ symmetry.

[46] H. S. J. van der Zant, F. C. Fritschy, W. J. Elion, L. J. Geerligs, and J. E. Mooij, Phys. Rev. Lett. 69, 2971 (1992).

[47] D. D. Solnyshkov, R. Johne, I. A. Shelykh, and G. Malpuech, Phys. Rev. B 80, 235303 (2009).

[48] See Supplemental Material 5 at [url] for resonant excitation simulations.

[49] P. Tsotsis, P. S. Eldridge, T. Gao, S. I. Tsintzos, Z. Hatzopoulos, and P. G. Savvidis, New Journal of Physics
14, 023060 (2012).

[50] M. Pasienski and B. DeMarco, Optics Express 16, 2176 (2008).

[51] A. P. D. Love, D. N. Krizhanovskii, D. M. Whittaker, R. Bouchekioua, D. Sanvitto, S. A. Rizeiqi, R. Bradley, M. S. Skolnick, P. R. Eastham, R. André, and L. S. Dang, Phys. Rev. Lett. 101, 067404 (2008).

[52] H. L. Stover and W. H. Steier, Appl. Phys. Lett. 8, 91 (1966).

[53] E. Kammann, T. C. H. Liew, H. Ohadi, P. Cilibrizzi, P. Tsotsis, Z. Hatzopoulos, P. G. Savvidis, A. V. Kavokin, and P. G. Lagoudakis, Phys. Rev. Lett. 109, 036404 (2012) 